\title{
Poly-Wire-Coupled Single Crystal Silicon HARPSS Micromechanical Filters Using Oxide Islands
}

\author{
Reza Abdolvand, Gavin K. Ho, and Farrokh Ayazi \\ School of Electrical and Computer Engineering, Georgia Institute of Technology \\ Atlanta, GA 30332-0250
}

\begin{abstract}
This paper reports on the implementation of single crystal silicon micromechanical filters coupled through a thin polysilicon wire using a SOI-based HARPSS process. The formation of suspended thin poly wires, which provide weak mechanical coupling between high quality factor thick microresonators, are enabled by the use of thick sacrificial oxide islands created between adjacent single crystal silicon resonators. The oxide islands are also used beneath the input and output pads of the filters to reduce the parasitic capacitances to substrate. Mechanical coupling of microresonators using thin-film wires provides superior design flexibility since the material and thickness of the coupling element can be chosen independent of those of the resonators. Measurement results obtained from $800 \mathrm{kHz}$ two-pole HARPSS micromechanical filters with thin poly wire are presented.
\end{abstract}

\section{INTRODUCTION}

Previously reported micromechanical filters have shown high selectivity, narrow bandwidth, and low insertion loss in the range of low to high frequency [1-3]. The bandwidth of these filters is primarily determined by the stiffness ratio of the coupling element to resonator [4]. To date, a few implementations of mechanically-coupled resonator filters have been reported using polysilicon surface micromachining technology, in which the vibrating resonator and the coupling element thickness is limited by the deposition thickness of the thin film polysilicon (typically a few microns).

The mixed-mode HARPSS fabrication technology has the ability to create sub-100nm vertical capacitive gaps along tens of microns thick high-Q single crystal silicon (SCS) resonators $[\mathbf{5 , 6 ]}$. This in turn provides stronger capacitive transduction and lower motional resistance. Electrically-coupled micromechanical filters have been demonstrated using HARPSS resonators [7,8]; however, mechanical coupling of laterally-vibrating SCS HARPSS resonator was not demonstrated in the past. Figure 1 illustrates two ways of coupling SCS resonators through axially loaded coupler beams. In Fig. 1a, the coupler has the same thickness as the resonator structures, while in Fig. 1b the coupler is a thin wire such as a surface-deposited beam. One of the main challenges in implementing narrow bandwidth filters is providing a small coupling strength between constituent resonators. Thick coupling elements have large spring constants and consequently wider filter bandwidth. Reducing the width of the coupling element to decrease the stiffness is limited by lithography and long compliant couplers may cause excessive mass loading. Thin coupling wires present a viable solution to narrow bandwidth filtering, if they can be easily fabricated. Both the axial and flexural stiffness of a beam are directly proportional to its thickness (i.e. height), which can be greatly reduced in a thin-film deposition process. Therefore, compliant thin-film wires have great potential for resonator coupling in narrow bandwidth filters and is the main focus of this work (Fig. 1b).

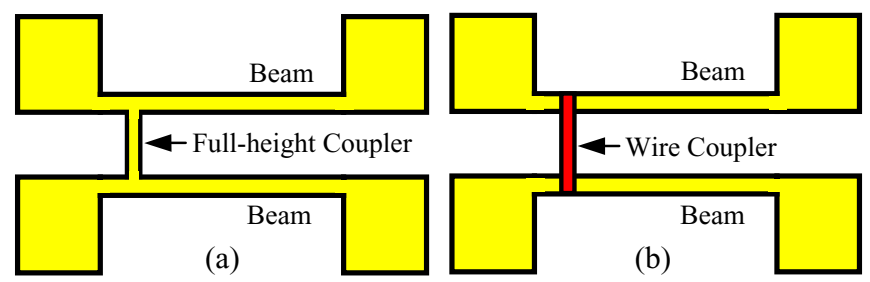

Figure 1. Coupling of two HARPSS beam resonators via a) fullheight silicon beam; and b) surface-deposited beam (e.g. poly wire)

The resonance frequencies and mode shapes for the flexural modes of a beam resonator are:

$$
\begin{gathered}
f_{n}=\frac{\beta_{n}{ }^{2} w}{4 \sqrt{3} \pi} \sqrt{\frac{E}{\rho}} \\
\phi_{n}(x)=C_{n}\left[\sinh \beta_{n} x-\sin \beta_{n} x+\alpha_{n}\left(\cosh \beta_{n} x-\cos \beta_{n} x\right)\right]
\end{gathered}
$$

where $w$ is the width of the beam, $E$ is the Young's modulus, $\rho$ is the density, and $\beta_{n} L=4.7300,7.8532,10.995$ for the first three modes [9]. Modeling the beam resonator as a single degree of freedom system for the fundamental flexural mode, $\omega_{o}=2 \pi f_{o}=\sqrt{k_{\text {eff }} / m_{\text {eff }}}$ where the effective mass $m_{\text {eff }}$ and effective stiffness $k_{\text {eff }}$ are evaluated at a particular point of interest. For a 1D homogeneous continuous system, these parameters are:

$$
m_{e f f}=\frac{K E_{t o t}}{(1 / 2) \dot{\phi}_{c}^{2}}=\rho w h \frac{\int_{L} \phi^{2}(x) d x}{\phi_{c}^{2}}, \quad k_{e f f}=\omega_{o}{ }^{2} m_{e f f}
$$

where $\phi_{c}$ and $\dot{\phi}_{c}$ are the modal displacement and velocity at $x=x_{c}$. In a lumped model of a two-pole mechanically-coupled bandpass filter with a coupling stiffness $k_{c}$ (Fig. 2), the frequency separation between the resonances is approximated by:

$$
\Delta f=f_{2}-f_{1} \approx f_{1} \frac{k_{c}}{k_{e f f}}
$$

A large $k_{\text {eff }}$ can be obtained via low-velocity coupling [2], where the modal displacement $\phi_{c}$ is small. It is therefore advantageous to place coupling element closer to the resonator clamped boundary.
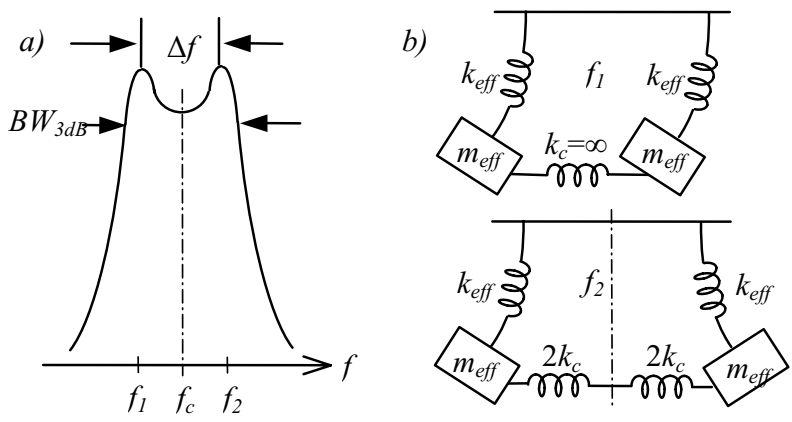

Figure 2. a) Frequency response of a two-pole filter, b) vibration modes of a discrete $2 \mathrm{DOF}$ mechanical system.

Travel support has been generously provided by the Transducers Research Foundation and by the DARPA MEMS and DARPA BioFlips programs. 


\section{FABRICATION PROCESS}

In this work, the SOI-based HARPSS process is augmented with thick silicon dioxide islands to enable formation of suspended thin poly wires. Placement of such thick oxide islands beneath the input and output wire-bonding pads has the added benefit of substantially reducing the parasitic capacitances to the substrate.

The process begins on an SOI wafer with a $5-50 \mu \mathrm{m}$ thick device layer. The first step involves etching narrow trenches for isolation and oxide island formation. Numerous $1.5 \mu \mathrm{m}$ wide trenches are etched on a $1.5 \mu \mathrm{m}$ pitch into the SOI device layer (Fig. $3 a$ ), followed by subsequent thermal oxidation of the wafer to consume the remaining silicon and create the thick silicon dioxide islands. To ensure formation of voidless oxide islands, a $0.5 \mu \mathrm{m}$ LPCVD oxide layer is then deposited, followed by plasma etch back of the oxide on the surface (Fig. 3b).

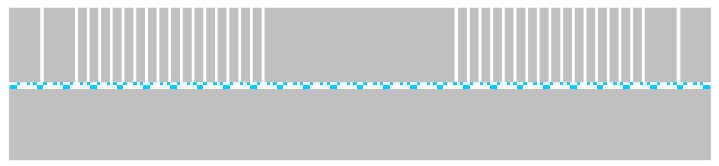

a) Trench etching

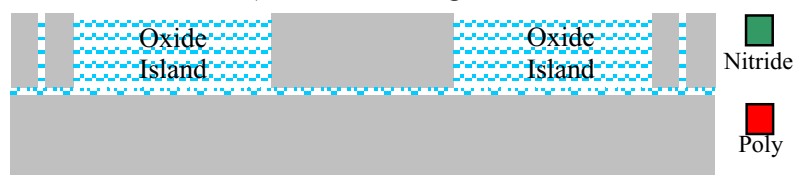

b) Oxidation, oxide deposition and etch back

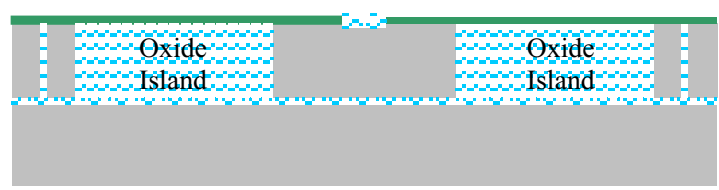

c) Nitride deposition/patterning

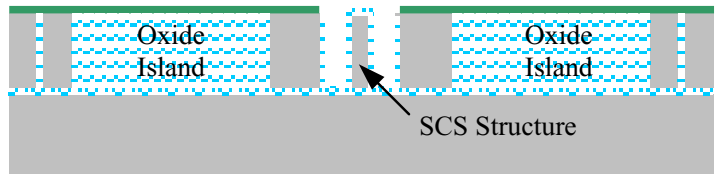

d) Trench etching and sacrificial oxide growth

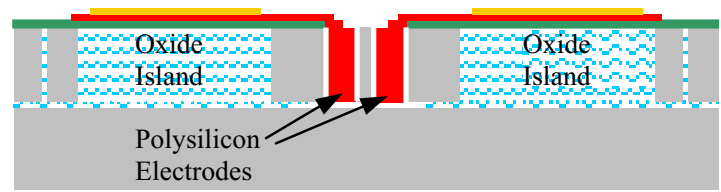

e) Two poly depositions and patterning, gold deposition and patterning, and HF release

Figure 3. Fabrication process flow of the SOI-based HARPSS process with thick oxide islands.

To protect the solid oxide islands from the final HF release of the device, a $0.4 \mu \mathrm{m}$ LPCVD nitride layer is deposited and patterned to cover the oxide (Fig. 3c). After this, $5 \mu \mathrm{m}$ wide trenches are etched into the silicon device layer and subsequently filled with thin layers of oxide and polysilicon. The thickness of the thin sacrificial oxide defines the capacitive transduction gaps (Fig. 3d). The polysilicon layer is patterned on the surface to form the poly pads and coupling wires. In the next step, a thin layer of gold is deposited on top of polysilicon pads through a lift-off process. The last lithography step is performed to etch polysilicon inside the trenches and define electrodes. Finally, the device is released in $\mathrm{HF}: \mathrm{H}_{2} \mathrm{O}(1: 1)$ solution (Fig. 3e). Figure 4 shows a cross section of a smooth polysilicon bonding pad with a solid oxide island underneath implemented on a $15 \mu \mathrm{m}$ thick SOI substrate.

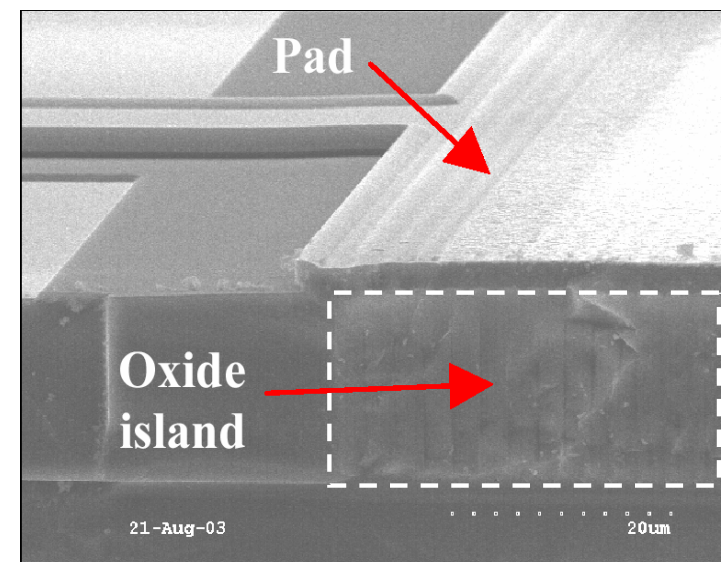

Figure 4. Cross-section of a bonding pad and the solid oxide island created in the $15 \mu \mathrm{m}$ thick silicon device layer of an SOI wafer.

\section{Suspended Poly Wire}

A polysilicon coupling wire can be incorporated into this modified version of the HARPSS process without any additional processing steps. The oxide islands, used for reduction of the pad capacitance, are also created between adjacent resonators for this purpose (Fig. 5a). In a second poly deposition and patterning step, a poly wire is patterned on the oxide island, which connects the neighboring resonators at the top surface (Fig. 5b). Since these oxide islands are not protected with nitride, they are etched in the final HF release step, and a suspended coupling wire is created (Fig. 5c).

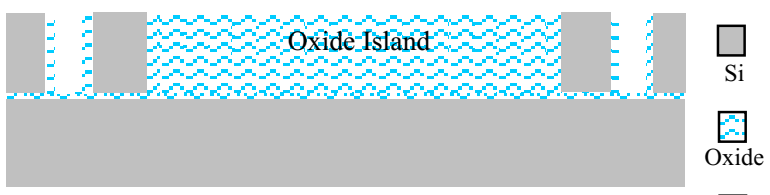

a) Oxide island placement between two beams

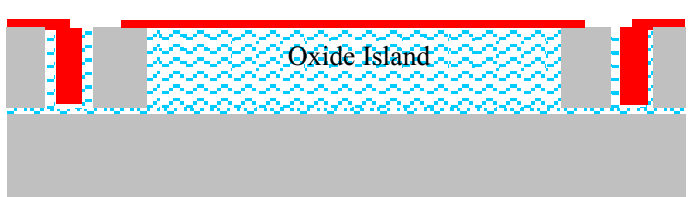

b) Poly deposition and patterning

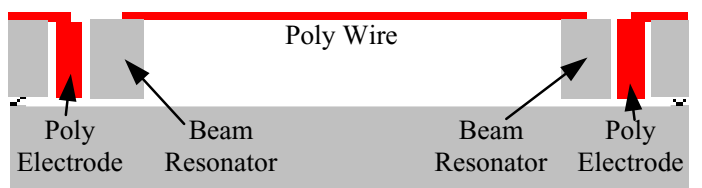

c) Oxide etch (HF release)

Figure 5. Fabrication process for formation of the poly wire

In this process, polysilicon was used for the coupling wire to minimize the number of mask layers. However, any other process compatible material (e.g. HF resistant metals) can be used to make the wire. Figure 6 shows an SEM of an electromechanical HARPSS filter consisting of two $300 \mu \mathrm{m}(l) \times 9 \mu \mathrm{m}(w) \times 15 \mu \mathrm{m}(h)$ single crystal silicon clamped-clamped beams coupled with a suspended poly wire centered at $10 \mu \mathrm{m}$ from the clamped boundary. Figure 7 is the close up of the poly wire and $15 \mu \mathrm{m}$ thick SCS 
beams. Figure 8 is the SEM of a HARPSS filter with similar SCS beams coupled with a full-height SCS beam.

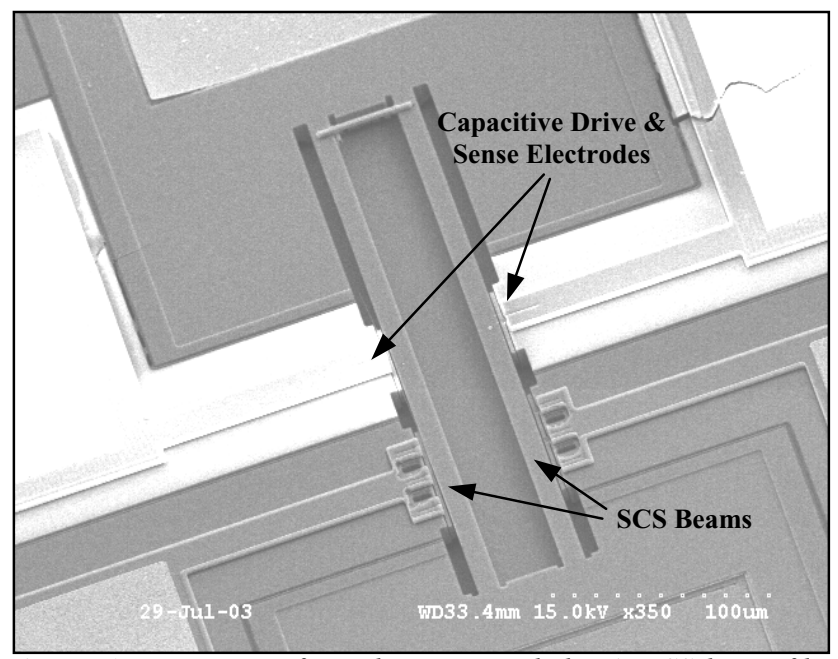

Figure 6. Top view of a poly-wire-coupled HARPSS beam filter consisting of two $300 \mu \mathrm{m} \times 9 \mu \mathrm{m} \times 15 \mu \mathrm{m}$ beams. The poly-wire is centered at $10 \mu \mathrm{m}$ from the beam resonator support.

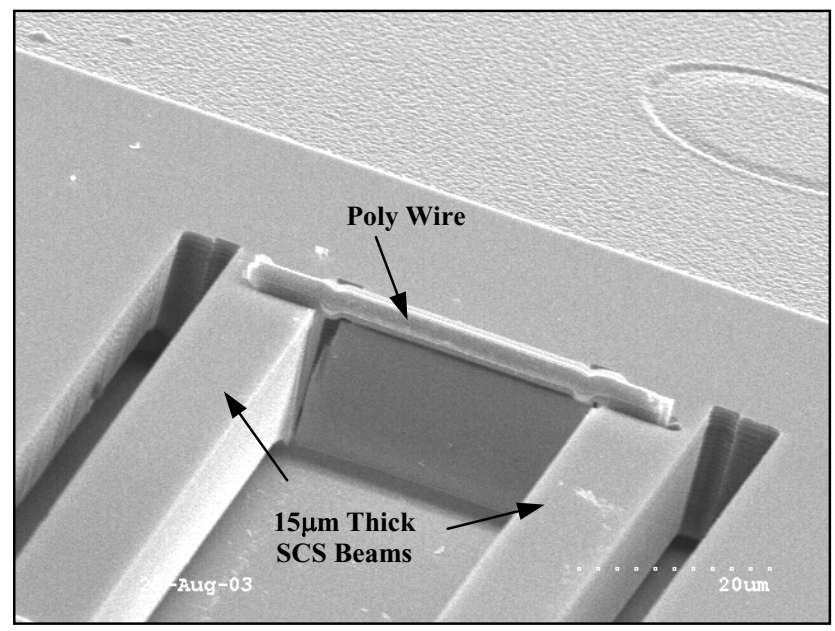

Figure 7. Close up view of the poly wire of Fig. 6.

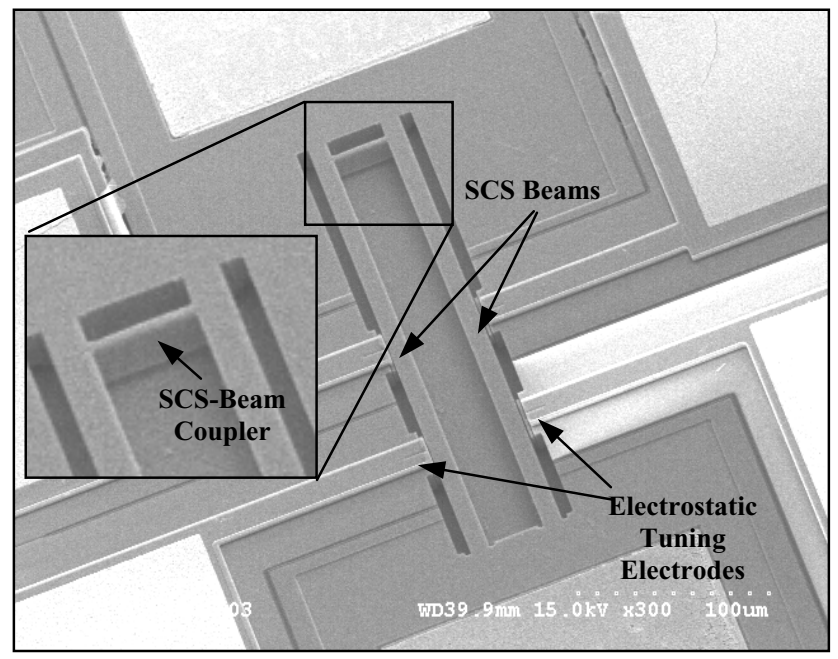

Figure 8. SEM picture of a fabricated HARPSS beam filter with a full-height SCS beam coupler

\section{EXPERIMENTAL RESULTS \& ANALYSIS}

The specifications of the devices in Figs. 6 and 8 are listed in Table 1 . Their frequency responses were measured under vacuum and are shown in Figs. 9a and $9 \mathrm{~b}$ respectively. The frequency separation between two resonance peaks in the poly-wire-coupled device $(12 \mathrm{kHz})$ is 3 times lower than that of the SCS-beamcoupled device $(35 \mathrm{kHz})$. Since the dimensions of the constituent resonators and the coupling locations are the same in both devices, the implementation with the poly-wire coupling element is clearly advantageous for reducing the frequency separation. The measured $Q$ for each individual peak in Fig. 9 is 7300 .

Table 1. Poly-wire-coupled and full-height SCS-beam-coupled device dimensions

\begin{tabular}{|l|c|c|}
\hline \multicolumn{1}{|c|}{ Parameter } & Poly-Wire & SCS-Beam \\
\hline Resonator $l \times w \times h[\mu \mathrm{m}]$ & $300 \times 9 \times 15$ & $300 \times 9 \times 15$ \\
\hline Coupler Material & Polysilicon & SCS \\
\hline Coupler Modulus & $150 \mathrm{GPa}$ & $168 \mathrm{GPa}$ \\
\hline Coupler $l \times w \times h[\mu \mathrm{m}]$ & $30 \times 2 \times 3$ & $30 \times 2 \times 15$ \\
\hline Coupling Dist. from Beam End & $10 \mu \mathrm{m}$ & $10 \mu \mathrm{m}$ \\
\hline
\end{tabular}
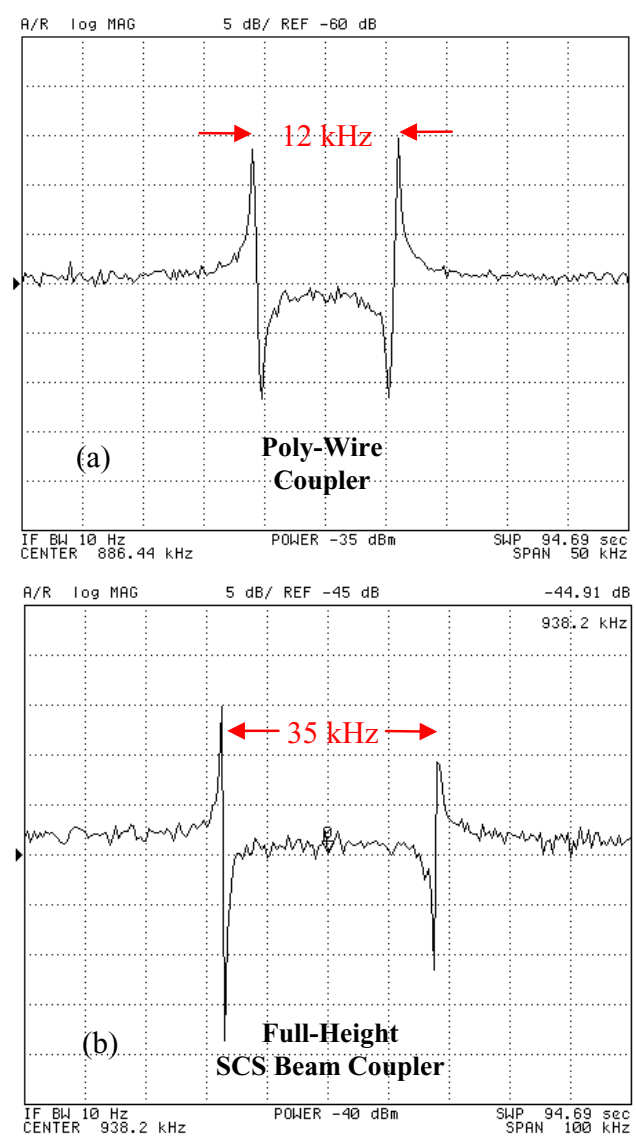

Figure 9. Frequency response plots measured for a) the poly-wirecoupled device of Fig. 6, and b) the SCS-beam-coupled device of Fig. 8 .

For the device dimensions in Table 1, the effective mass $m_{\text {eff }}$ at the coupling location for both devices were found to be 1800 times the resonator mass using Equation 4. Noting that $m_{\text {eff }}$ is much larger than the mass of the coupling element $m_{c}$, mass loading is negligible. In the out-of-phase mode, the coupling beam is axially 
loaded, so the stiffness and effective mass of the coupling element are

$$
k_{c}=\frac{E_{c} w h}{L} \text { and } m_{c, e f f}=\frac{m_{c}}{8} .
$$

The coupling strength $k_{c}$ is proportional to the height of the coupling beam and the advantage of using thin poly-wires for coupling is evident. Substituting Equation 6 with specifications in Table 1 into Equation 3, the calculated $\Delta f$ are $3.4 \mathrm{kHz}$ and $28 \mathrm{kHz}$ for the poly-wire- and SCS-beam-coupled devices respectively. The analytical results are consistently smaller than experimental data, indicating that through-support coupling [3] which was not included in this model provides additional coupling in this topology.

To obtain a filter response from the poly-wire-coupled device, the motional resistance was reduced by applying polarization voltages of $65 \mathrm{~V}$, and external termination resistances were placed at both the input and output. Figure 10 is the filter response obtained with resistive termination.

The electrostatic tuning electrodes on each beam (Fig. 8) offer a tuning mechanism to alter the resonator frequency and compensate for the fabrication non-idealities. The minimum coupling-limited frequency separation occurs when the two resonators are matched [1]. The variation in the frequency separation of the poly-wire coupled filter is shown in Fig. 11.

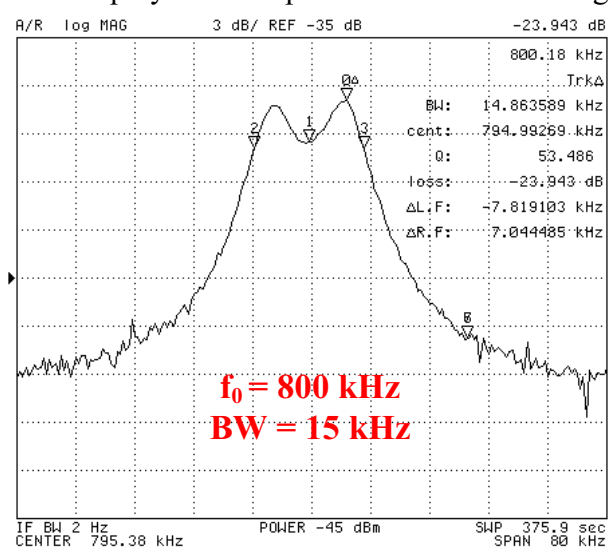

Figure 10. Filter characteristic from an array of two $300 \mu \mathrm{m} \times$ $10 \mu \mathrm{m} \times 15 \mu \mathrm{m}$ poly-wire-coupled beams.

\section{CONCLUSIONS}

The structural coupling of adjacent laterally-excited micromechanical resonators using a coupling element of a different material to enable narrow bandwidth filters was demonstrated.
Thick oxide islands incorporated into a new HARPSS process enabled fabrication of a thin suspended polysilicon wire with a good structural bond to the single crystal silicon resonators. This wire-fabrication technology is applicable for coupling other resonator geometries and has potential for minimally-invasive resonator anchoring schemes.

\section{ACKNOWLEDGMENTS}

The authors wish to thank Siavash Pourkamali and the cleanroom staff of the Georgia Tech Microelectronics Research Center for their assistance. This work was funded under the DARPA NMASP program.

\section{REFERENCES}

1. K. Wang and C. T.-C. Nguyen, "High-order medium frequency micromechanical electronic filters", IEEE Jnl. of Microelectromechanical Systems (JMEMS), V8, N4, (1999), pp. 534-557.

2. K. Wang, F. D. Bannon III, J. R. Clark, and C. T.-C. Nguyen, "Q-Enhancement of Microelectromechanical Filters Via LowVelocity Spring Coupling," Proc. of 1997 IEEE Ultrasonics Symp, (1997), pp. 323-327.

3. G. K. Ho, R. Abdolvand, and F. Ayazi, "Through-SupportCoupled Micromechanical Filter Array," Proc. IEEE MEMS'04 Conf., Maastricht, The Netherlands (2004), pp. 769-772.

4. R. A. Johnson, Mechanical Filters in Electronics, 1983.

5. S. Pourkamali, A. Hashimura, R. Abdolvand, G. Ho, A. Erbil, and F. Ayazi, "High-Q Single Crystal Silicon HARPSS Capacitive Beam Resonators with Sub-micron Transduction Gaps," IEEE Journal of Microelectromechanical Systems, Vol. 12, No. 4, August 2003, pp. 487-496.

6. S. Pourkamali and F. Ayazi, "SOI-Based HF and VHF SingleCrystal Silicon Resonators with Sub-100 Nanometer Vertical Capacitive Gaps," Tech. Digest 12th Int'l Conf. on Solid State Sensors, Actuators and Microsystems (Transducers'03), Boston MA (2003), pp. 837-840.

7. S. Pourkamali, R. Abdolvand, and F. Ayazi, "A $600 \mathrm{kHz}$ Electrically Coupled MEMS Bandpass Filter," Proc. IEEE Int'l MEMS Conf. (MEMS‘03), Kyoto, Japan (2003), pp. 702-705.

8. S. Pourkamali, R. Abdolvand, G. K. Ho, and F. Ayazi, "Electrostatically Coupled Micromechanical Beam Filters", Proc. of IEEE MEMS'04 Conf., The Netherlands (2004), pp. 584-587.

9. S. S. Rao, Mechanical Vibrations, 3E, 1995, Addison Wesley, Reading MA, pp. 526-527.

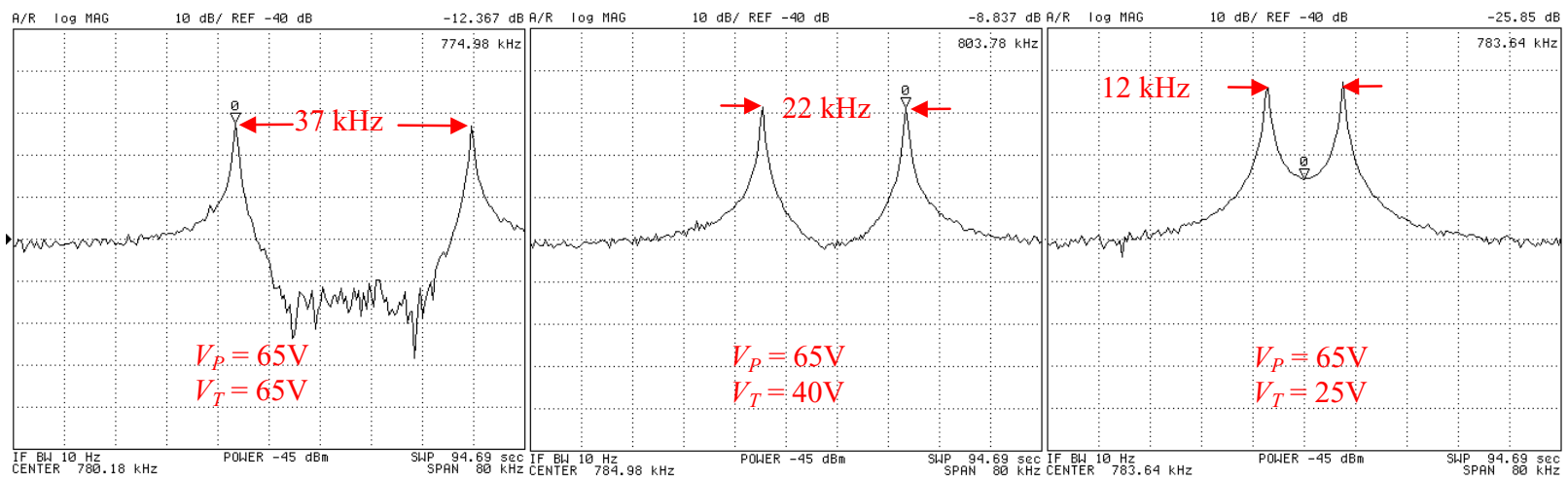

Figure 11. Electrostatic tuning for frequency matching to produce a coupling-limited filter bandwidth ( $V_{T}$ is applied to one tuning electrode with the remaining tuning electrode grounded). A polarization voltage $V_{P}$ of $65 \mathrm{~V}$ was applied to the body of the resonators. 